\title{
Operation Status Monitoring of Transmission Tower in Power System based on Data Fusion
}

\author{
Haiting Ji, Jianfeng Liu
}

\author{
Chongqing Key Laboratory of Geological Environment Monitoring and Disaster \\ Early-Warning in Three Gorges Reservoir Area, Chongqing Three Gorges University, \\ Chongqing, China
}

Received: June 8, 2021. Revised: December 19, 2021. Accepted: January 13, 2022. Published: January 14, 2022.

\begin{abstract}
This paper studies the application of data fusion technology in power system to solve some difficult problems in this complex energy system. A transmission tower identification and bird nest detection method based on corner, line, color and shape features is proposed. Through LSD (Line Segment Detection) and Harris corner detection method, the straight line segment and corner point in the image are extracted respectively. Combined with triangle method, the actual tilt angle of tower is measured; According to the nesting rule of birds in transmission towers, the basic unit segmentation algorithm of transmission towers is proposed, and the basic unit segmentation of transmission towers is realized by using the local maximum of the target pixel row statistical histogram. The algorithm proposed in this paper can effectively solve the problems of on-line measurement of tilt angle of transmission tower and on-line detection of bird's nest, which will lay a theoretical foundation for on-line monitoring of transmission tower status.
\end{abstract}

Keywords-Data fusion, Electric power system, Transmission tower, Condition monitoring

\section{INTRODUCTION}

$\mathrm{E}$ lectric energy has become an indispensable product in daily life and industrial production, and the stability of electric energy transmission has an increasing influence on social development. As an important part of electric energy transmission system, the running state of transmission tower plays a vital role in the stability of electric energy transmission [1]. The inclination of transmission tower will weaken the stability of the tower, which will eventually lead to the collapse of the tower and cause serious power failure. The existence of bird nest on the tower will increase the frequency of bird activities near the tower, and also increase the probability of power system failure, which will destroy the stability of power transmission and seriously affect the social and economic development [2-3]. Therefore, it is very necessary to monitor the running status of transmission towers on line.

With the construction of smart grid and ultra-high voltage grid, various transmission lines with different voltage levels are increasing, which requires more and more transmission towers to be built. Literature [4] uses the methods of residual sensitivity and innovation vector to correct the power grid operating parameters. However, the power grid operating conditions change in real time, which makes the real-time estimation of power system parameters closer to the actual power grid operation. It is particularly important to take dynamic parameters into the estimation of power grid operating conditions. Literature [5] analyzes the influence of PMU (phasor measurement unit) measurement value on power system state estimation. Literature [6] gives the linear measurement equation of power system in real number form based on rectangular coordinate system and the corresponding linear static state estimation algorithm, but it does not fully integrate the measurement information of SCADA (Supervisory Control and Data Acquisition) system. Literature [7-8] puts forward the application and development prospect of state estimation based on synchronous measurement, and dynamically monitors the system through state estimation. Literature [9-11] puts forward a state estimation model of hybrid measurement after PMU is installed in some nodes on the basis of traditional SCADA measurement system, which improves the accuracy of power system state estimation. However, these methods are still static state estimation because they are also based on the measurement information of the same section.

Data fusion technology refers to making full use of the characteristics of multiple knowledge sources and sensors, adopting various information processing methods, combining data collected by different knowledge sources or sensors according to established rules, and extracting effective information of objects, so as to form a more comprehensive, reliable and complete description of the information of a certain processing object [12]. Through the above research, we have developed a complete monitoring system for transmission towers based on data fusion, and through the test operation, it has been verified that the system can run safely 
and stably, and can obtain accurate and reliable measurement data, thus realizing the function of online inclination monitoring for towers.

\section{RESEARCH METHOD}

\section{A. Decentralized Coordination Control Decision of Power} System based on Data Fusion

There are three basic fusion types in the hierarchical structure of information fusion, namely, data fusion in data layer, data fusion in feature layer and data fusion in decision layer [13].

On the basis of D-S evidence theory and Dempster merging rule [14-15], chapter introduces a modified method which can be used for relevant evidence fusion, and applies this method to the type discrimination of coupled subsystem model in power system.

In the process of evidence acquisition, every evidence source $E$ appears, one or more new focal elements $A_{i}$ will be generated, and the unknown information $m(\Theta)$ will be reduced. On the one hand, the amount of information provided by this evidence source is related to the basic credibility $m\left(A_{i}\right)$ of $A_{i}$, on the other hand, it is also related to the cardinal number $\left|A_{i}\right|$ of $A_{i}$. The number of focal elements is $n\left(A_{i}\right)$, and the strength $S(E)$ of evidence can be defined as follows.

$$
S(E)=\sum_{i=1}^{n\left(A_{i}\right)} \frac{m\left(A_{i}\right)}{\left|A_{i}\right|}
$$

For any $i$, if $m\left(A_{i}\right)=0$, that is, the maximum unknown information $m(\Theta)=1$, then $S(E)=0$, the evidence $E$ is empty and does not contain any useful information. If $\left|A_{i}\right|=1$ and $m(\Theta)=0, S(E)=1$ and $E$ contain the most useful information, so $S(E) \in[0,1]$.

There are two evidences $E_{1}, E_{2}$, whose basic reliability is $m_{1}, m_{2}$ and focal element is $A_{i}, A_{j}$. If they are related, this correlation will be reflected in some focal elements, that is, some focal elements in $E_{1}$ and some focal elements in $E_{2}$ are produced by the same evidence source. This focal element is called related focal element, which is recorded as $R_{i j}$, its basic credibility is $m_{d}$, and the number of related focal elements is $n\left(R_{i j}\right)$, which is defined by the evidence intensity. The evidence correlation intensity is as follows.

$$
S\left(E_{1}, E_{2}\right)=\sum_{i, j=1 R_{i j} \neq \Theta}^{n\left(R_{i j}\right)} \frac{m_{d}\left(R_{i j}\right)}{\left|R_{i j}\right|}
$$

When $S\left(E_{1}, E_{2}\right)=0$, the evidence $E_{1}, E_{2}$ is independent of each other; $S\left(E_{1}, E_{2}\right)=1$ and $S\left(E_{1}\right)>S\left(E_{2}\right)\left(\right.$ or $S\left(E_{1}\right)<S\left(E_{2}\right)$ ) indicate that $E_{1}$ contains $E_{2}$ (or vice versa), so $S\left(E_{1}, E_{2}\right)$ is used to describe the correlation between evidences.

If the actual test value $x_{j}(j=1 \sim 4)$ of the $i(i=1,2, \cdots, m)$-th evidence body satisfies Gaussian distribution, the prior probability is

$$
p\left(x_{j} \mid A_{j}\right)=\frac{1}{\sqrt{2 \pi \sigma_{i}}} \exp \left[\frac{1}{2}\left(\frac{x-\mu_{i}}{\sigma_{i}}\right)^{2}\right]
$$

D-S evidence theory is a decision-making theory, which combines multiple evidences to make decisions, and makes a reasonable information theory interpretation of reasoning. D-S evidence theory requires that when constructing the basic reliability allocation function, the probability measure expressed by $[0,1]$ interval cannot be allocated to all possible events, otherwise there is no uncertain part, which degenerates into a probability problem [16]. Based on the above idea, the reliability function is constructed as follows for the specific problems of property model identification:

The basic credibility given to the property model $A_{j}$ by the $i$-th evidence body is

$$
m_{i}\left(A_{j}\right)=\frac{C_{i}\left(A_{j}\right)}{\sum_{j=1}^{4} C_{i}\left(A_{j}\right)+\alpha\left(A_{j}\right)\left(1-R_{i}\right)\left(1-\alpha_{i} \beta_{i}\right)}
$$

The uncertainty probability value of the $i$-th evidence body is

$$
m_{i}(\Theta)=\frac{\alpha\left(A_{j}\right)\left(1-R_{i}\right)\left(1-\alpha_{i} \beta_{i}\right)}{\sum_{j=1}^{4} C_{i}\left(A_{j}\right)+\alpha\left(A_{j}\right)\left(1-R_{i}\right)\left(1-\alpha_{i} \beta_{i}\right)}
$$

Therefore, when calculating the uncertainty probability $m_{i}(\Theta)$ of evidence body, we mainly consider the unreliability factor $\left(1-R_{i}\right)$ of evidence body itself, the unreliability factor $\left(1-\alpha_{i} \beta_{i}\right)$ between evidence body and target category, and the similarity degree $\alpha\left(A_{j}\right)$ of evidence body measurement.

\section{B. Structure of Software and Hardware of Monitoring} System

On-line monitoring system for anti-toppling of transmission pole tower is composed of software and hardware, in which the hardware includes inclination measuring device composed of biaxial inclination sensor and GPRS communication module, and data acquisition system composed of GPRS receiving equipment and terminal PC [17]. The software is built by LabVIEW platform, and has the logical structure of login management, operation control and exit, in which the operation control includes three functions: tower real-time monitoring, data query and software management. On-line monitoring system software for anti-toppling of transmission line tower is based on Labview, which realizes the functions of synchronous acquisition, data query and data management. When the system is running, the tower inclination data sampled by the sensor is collected quickly and synchronously, and then processed and displayed, and the data is stored in the 
relevant database.

Inclination measuring device composed of two-axis inclination sensors, data transmission device composed of GPRS communication module and terminal system composed of terminal PC, which together complete the functions of real-time on-line monitoring of tower inclination and data transmission, processing, display and alarm. Fig. 1 shows the main structure of on-line monitoring system for anti-dumping of transmission pole tower.

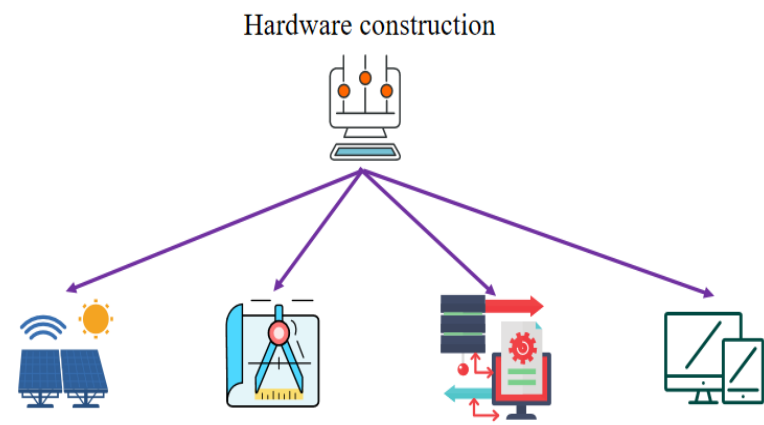

Solar power plant Solar power plant Data transmission Terminal system device

Figure 1. Main structure of on-line monitoring system for anti-dumping of transmission pole tower

Fig. 2 shows the main functions that need to be realized by the on-line monitoring software for anti-toppling of transmission pole tower. All the above functions can be realized by compiling with LabVIEW, and interfaces can be reserved for other monitoring modules, such as small weather monitoring, external force prevention video monitoring and so on.

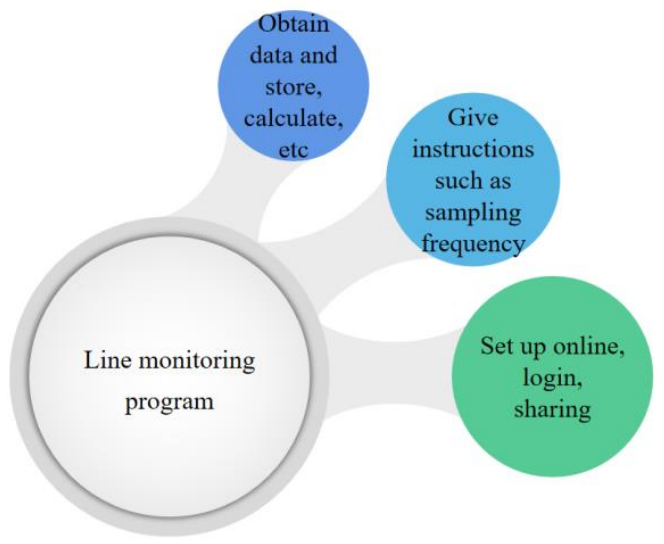

Figure 2. Main functional framework of detection software

\section{Transmission Tower Identification}

In image processing, straight lines represent certain structural features, while line segments are lower-level features compared with straight lines. The extraction of line features is of great significance for deeper image analysis [18]. LSD(Line Segment Detection), which has been included in OpenCV standard library, is the latest and best line segment detector. Line detection is different from straight line detection, which focuses on the bottom features, while straight line can be regarded as the abstraction of line segment, and straight line features can be regarded as middle features. Because it is closely related to line detection, it has attracted the close attention of many scholars who are concerned about line detection.

Compared with other LSD algorithms, LSD has the advantages of short operation time, no need to manually set parameters, no need for edge images and so on. Therefore, it has been widely praised in the research field. The treatment process is roughly as follows.

Step1. Firstly, the image is subjected to Gaussian downsampling operation to eliminate the sawtooth effect. And calculate the gradient value and gradient direction of each pixel in the image.

Step2. According to the gradient value, all points are pseudo-sorted, and the state list is established. All points are in unused initial state.

Step3. Generate a rectangle containing all the points that meet the conditions. The formula for judging the consistency of points is

$$
\left|\operatorname{region} \_\operatorname{ang}(t)-\operatorname{ang}(i, j)\right|<t
$$

In equation (6), region_ang $(t)$ is the direction angle of the $t$ -th region, and $\operatorname{ang}(i, j)$ is the gradient direction angle of the pixel $(i, j)$.

Step5. Judging whether the density of consistent points in the rectangle meets the threshold requirement, if not, cutting the rectangle into a plurality of rectangles, and repeating this step until the threshold requirement is met. The formula for calculating the density of consistent points is as follows.

$$
\rho=\frac{n}{N}
$$

In formula (7), $\rho$ is the density of consistent points, $n$ is the number of points satisfying the criterion of consistency, and $N$ is the total number of points in the rectangle.

Step6. Using a noise image whose pixel values are randomly assigned and do not contain straight line segments as a template, the assigned pixel values are independently and evenly distributed in $[0,2 \pi]$, and the $N F A$ value is calculated, which is defined as shown in Formula (8).

$$
N F A=N * P h 0[k(r, l) \geq k(r, i)]
$$

In which $N$ is the number of straight lines (rectangular boxes) in the current image, $k(r, l)$ is the number of consistent points in the $r$ rectangle in the template picture $l$, and $k(r, i)$ is the number of consistent points in the $r$ rectangle in the test picture $i$.

After the above steps, the linear steel structures of transmission towers have been detected, and there are also some non-tower background targets. The more significant the 
gray level change, the greater the corner quantity of the point [19-20].

Harris gives the analytical expression of gray scale change measure [21]:

$$
\begin{aligned}
& E(x, y)=\sum w(x, y)[I(x+u, y+v)-I(x, y)]^{2} \\
& =\sum w(x, y)\left[u I_{x}+v I_{y}+o \sqrt{u^{2}+v^{2}}\right]^{2}
\end{aligned}
$$

In the formula, $E(x, y)$ is the gray scale change measure in the window; $w(x, y)$ is the window function, Harris uses Gaussian function as the window, then $w(x, y)=e^{-\left(x^{2}+y^{2}\right) / \sigma^{2}}$; $(x, y)$ is the coordinates of pixels in the image window, $I$ represents the gray value of pixels, $I(x+u, y+v)$ is the gray value after moving, and $I(x, y)$ is the gray value before moving. Written in matrix form

$$
\begin{gathered}
\left.E(x, y) \approx[u, v] M \mid \begin{array}{l}
u \\
v
\end{array}\right] \\
M=\sum_{x, y} W(x, y)\left[\begin{array}{cc}
I_{x}^{2} & I_{x} I_{y} \\
I_{x} I_{y} & I_{y}^{2}
\end{array}\right]
\end{gathered}
$$

In this formula, matrix $M$ is the local autocorrelation matrix of this point, $I_{x}$ is the difference in $x$ direction, $I_{y}$ is the difference in $y$ direction, and $W(x, y)$ is Gaussian function.

Harris's corner response function $C R F$ expression is thus obtained

$$
C R F(x, y)=\operatorname{det}(M)-k\left(\operatorname{trace}(M)^{2}\right)
$$

In which, $\operatorname{det}(M)$ represents the determinant of matrix $M$ and $\operatorname{trace}(M)$ represents the trace of matrix. When the $C R F$ value of a target pixel is greater than a given threshold, the pixel is a corner.

After the above steps, the connection points between the steel structures of transmission towers are detected.

After obtaining the facies result map, the tower area is roughly divided, but there are holes and breaks, so it is necessary to extract the tower area completely through morphological reconstruction. In this chapter, a cascaded reconstruction filter is used, which achieves the purpose of reconstruction of transmission towers through multiple cascaded continuous processing of opening and closing operations. The binary images of transmission towers are repeatedly opened and closed by using gradually increasing structural elements.

After morphological reconstruction, the tower area has already appeared. Further, the initial positioning of transmission tower can be realized by labeling connected domain and judging the area [22]. The process is shown in Figure 3.

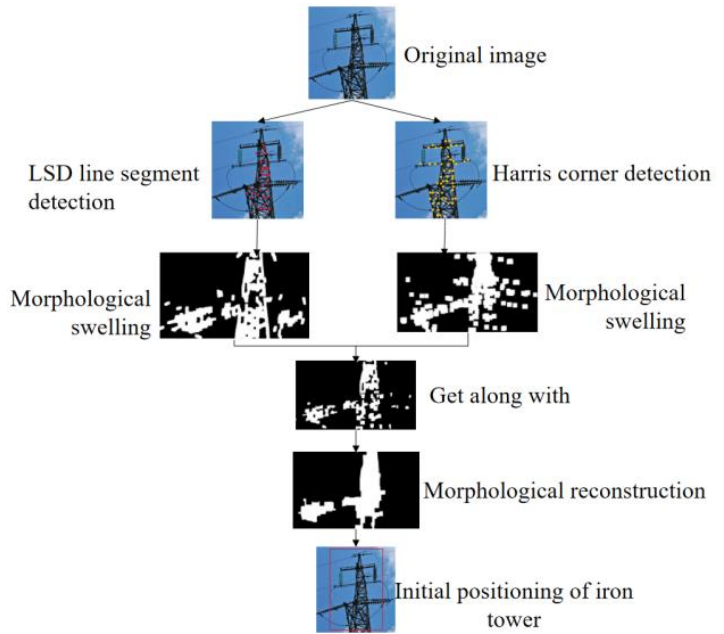

Figure 3. Flow chart of initial positioning of transmission tower

\section{Stability Monitoring of Transmission Tower}

It is necessary to collect the video images of the transmission tower along the line direction and the vertical line direction respectively, and the two collected positions should form a right triangle relationship with the tower, in which the tower is located at the right-angle vertex of the triangle.Hough line detection algorithm is used to obtain the projection of the geometric center line of the tower in two directions and calculate the inclination angle of the projection, and then the actual inclination angle of the tower is calculated according to the triangle method. Fig. 4 is a schematic diagram of triangle method.

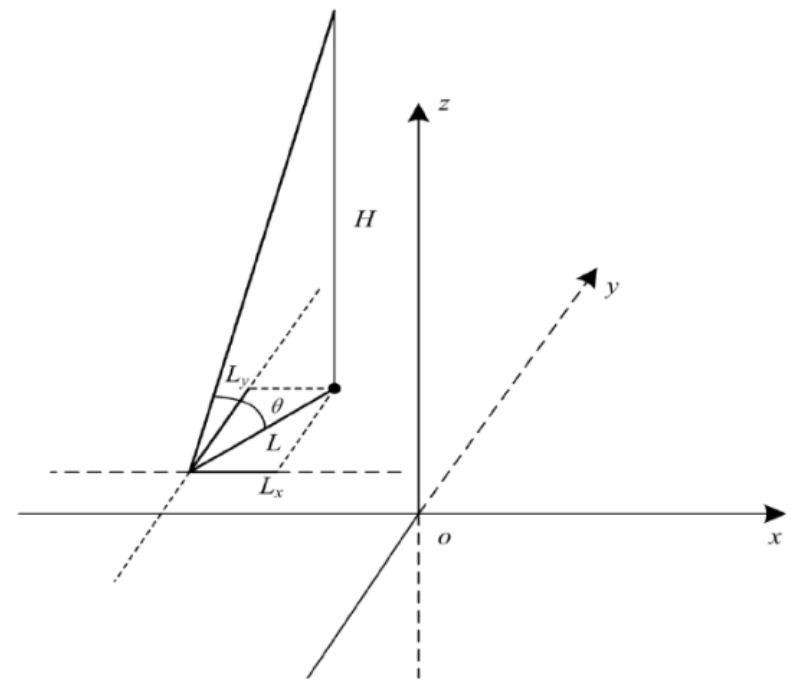

Figure 4. Schematic diagram of triangle method

The geometric center line of the tower is projected as $L$ on xoy plane, $L_{x}$ in $x$ axis direction and $L_{y}$ in $y$ axis direction, thus

$$
L^{2}=L_{x}^{2}+L_{y}^{2}
$$

Then the cotangent of the inclination angle projected by the geometric center line of the tower along the line direction 


$$
\cot \theta_{1}=\frac{L_{y}}{H}
$$

The cotangent of the inclination angle projected in the vertical line direction

$$
\cot \theta_{2}=\frac{L_{x}}{H}
$$

And the residual cutting of the actual inclination of the tower is

$$
\cot \theta=\frac{L}{H}
$$

Therefore, the actual tilt angle of the tower can be obtained

$$
\theta=\operatorname{arccot} \sqrt{\left(\cot \theta_{1}\right)^{2}+\left(\cot \theta_{2}\right)^{2}}
$$

It is necessary to collect the images of the transmission tower in the line direction and the vertical line direction respectively. The location of the two collections forms a right triangle relationship with the tower, and the tower is located at the right-angle vertex of the triangle.

Discrete wavelet transform is used to realize signal filtering. The continuous wavelet transform of signal $x(t)$ is defined as

$$
\begin{gathered}
W_{x}(j, n)=\int_{-\infty}^{\infty} x(t) \Psi_{j, n}^{*}(t) d t \\
\Psi_{j, n}(t)=2^{-j / 2} \Psi\left(2^{-j} t-n\right)
\end{gathered}
$$

In which $x(t)$ is the signal detected by each sensor; $\Psi_{j, n}(t)$ is the wavelet cardinality; $j$ is the scale factor; $n$ is the translation factor.

Preliminary stability diagnosis of transmission tower by RBF neural network method, and completes nonlinear mapping.

$$
f_{n}(e)=w_{0}+\sum_{i=1}^{n} w_{i} \phi\left(\left\|e-c_{i}\right\|\right)
$$

Generally take

$$
\phi\left(\left\|e-c_{i}\right\|\right)=\exp \left(-\frac{\left\|e-c_{i}\right\|^{2}}{\sigma_{i}^{2}}\right)
$$

Bayesian inference is used to fuse the preliminary diagnosis results, and the final fusion diagnosis results are obtained.

$$
\begin{gathered}
u(k)=\left\{\begin{array}{l}
+1, a_{0}(k)>0 \\
-1, a_{0}(k) \leq 0
\end{array}\right. \\
a_{0}(k)=\log \frac{P_{k}\left(H_{i} / u_{1}(k), \cdots u_{i}(k), \cdots, u_{n}(k)\right)}{P_{k}\left(H_{0} / u_{1}(k), u_{i}(k), \cdots, u_{n}(k)\right)}
\end{gathered}
$$

\section{RESUlt ANALYSIS AND DISCUSSION}

\section{A. Model Reliability Analysis}

A 4-machine coupling system is taken as an example to verify the effectiveness of the control scheme. The tie line from bus 11 to bus 12 is an important transmission line of $500 \mathrm{kV}$, which undertakes the main task of transmitting electric energy from the left area to the right area. In $0.1 \mathrm{~s}$, a three-phase short-circuit fault occurs at $20 \%$ of the distance between bus 11 and bus 12 from bus 11 , and the fault is removed for 0 . $4 \mathrm{~s}$, that is, the fault lasts for $0.3 \mathrm{~s}$..

The fault type here is three-phase short circuit fault; The fault line is the tie line from bus 11 to bus 12; The location is $20 \%$ away from the bus 11; The load near No.3 generator is a load with large fluctuation range of $30 \%$. All generators adopt excitation control mode, and the control target is stable power angle. There are 6 evidential bodies.

See fig. 5 for the reliability distribution function value and the fused trust function value of six evidences for four model properties of No.1 generator.

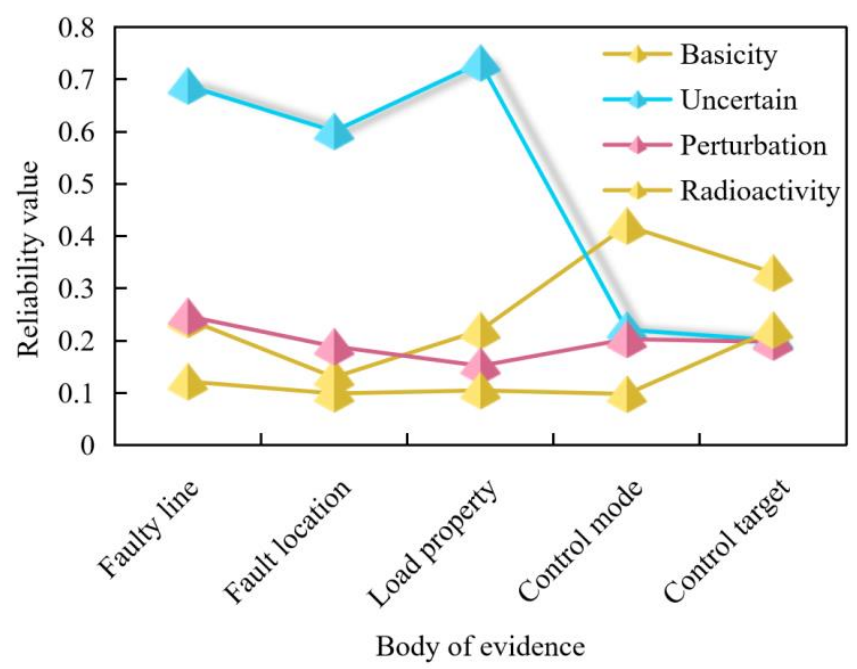

Figure 5. Reliability value of four models of No.1 generator

That the trust function value of the uncertainty of the No.1 generator model is the largest. According to the four principles of model identification, it can be concluded that the nature of the No.1 generator model is uncertain.

\section{B. Identification and Analysis of Transmission Tower}

In this section, algorithm experiments are carried out on aerial images of transmission towers collected by aerial photography of UAV patrol. The experiments are carried out in the software environment of 64-bit Windows 7, the hardware environment of E7500, 4G memory and 320G hard disk, and the development environment of Opencv2.4.10 and Microsoft visual studio 2010. The proposed methods of transmission tower identification and bird's nest detection are verified and analyzed. The algorithm of 600 aerial images with resolution of about $600 * 400$ pixels is tested, and some test results are shown in Figure 6. 

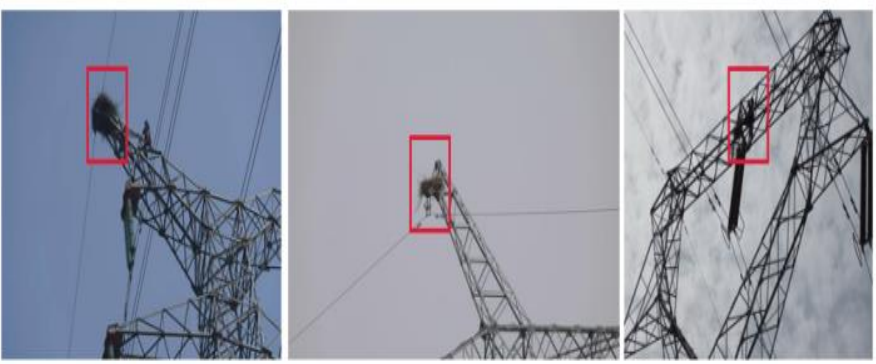

Figure 6. Partial test results

The purpose of the algorithm is to accurately identify and detect the anomalies of transmission towers and their nests in aerial images, so as to meet the needs of fault diagnosis using unmanned aerial vehicles. Since the existing research only focuses on tower identification, but does not detect the bird's nest in the tower, the algorithm in this paper is compared with the existing transmission tower identification algorithm. Firstly, the robustness of the algorithm is analyzed, and then the time consumption and detection rate of the algorithm are analyzed. Comparing the algorithm in this paper with the methods in reference [20] and reference [21], the average time consumption and detection rate of the algorithm are shown in Figure 7.

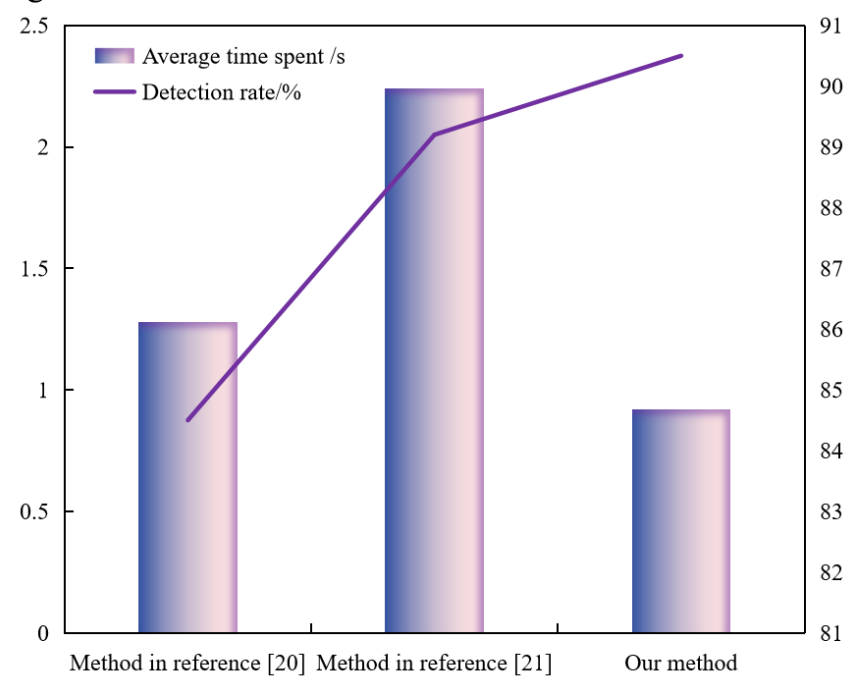

Figure 7. Comparison of average time consumption and detection rate of algorithm

Fig. 7 shows that the method in reference [21] takes a lot of time. Compared with the other two algorithms, the algorithm in this paper has the smallest average time consumption and the highest detection rate. Based on the performance comparison of the above three aspects, the proposed algorithm is robust, which meets the requirements of rapidity, and at the same time, the accuracy of transmission tower detection reaches over $92 \%$.

\section{Analysis of Tower Tilt Detection Algorithm}

Four transmission tower images with representative backgrounds are selected for verification, and the verification results are shown in Figure 8.

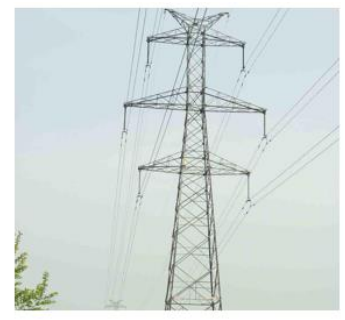

(a)

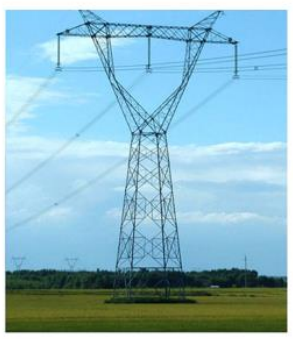

(c)

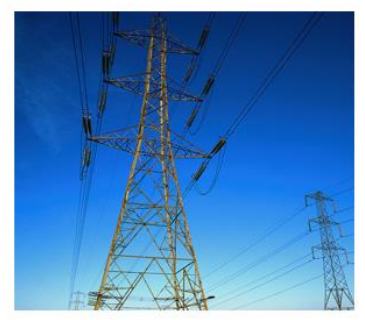

(b)

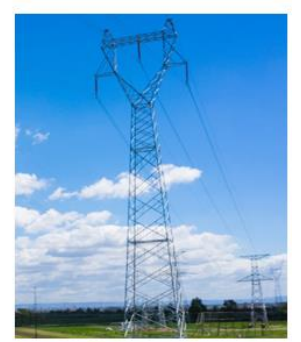

(d)
Figure 8 . Verify image

The verification results in Figure 8 show that the algorithm proposed in this chapter can process and calculate the transmission tower images with four typical backgrounds well, and has universal adaptability and strong robustness.

However, it is difficult to select and extract SIFT features of transmission tower images. Moreover, using advanced features of images to obtain tower tilt angle is slow and takes a long time to process [22]. In this paper, through in-depth observation and analysis of the geometric structure characteristics of the tower, the method of extracting the outer contour of the transmission tower and calculating the geometric center line of the outer contour is proposed. By calculating the inclination of the geometric center line along the line and perpendicular to the line and calculating the actual inclination of the tower by combining the triangle method, the calculation results are accurate, and the processing speed is fast and the processing time is short.

In this paper, the experimental images and four verification images are programmed, and the running time of the calculation and processing method proposed in reference [22] is verified. In addition, the running time of the tower tilt angle measurement method proposed in this paper is also verified by programming, and the running performance of the two methods is compared. The comparison results are shown in Figure 9. 


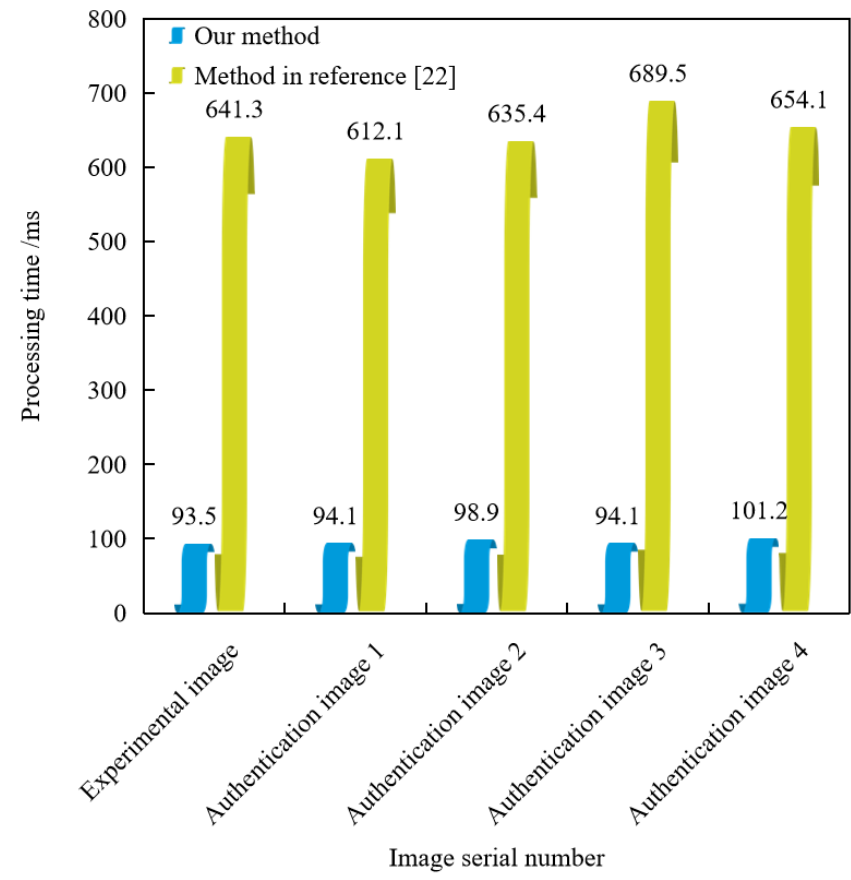

Figure 9. Performance comparison of two treatment methods

The data in fig. 9 are the processing time obtained by processing each image 10 times and averaging, so the method proposed in this paper is more suitable for real-time online measurement of transmission tower tilt angle.

To sum up, the triangle method proposed in this paper can measure the tilt of the tower on line in real time. Through the detection of the actual transmission tower image, the advantages of the method proposed in this paper are as follows. Optimizing pretreatment can reduce the interference of noise and unnecessary details, eliminate the influence of transmission lines and other towers on target tower contour detection in the background, and improve the detection accuracy. The acceleration sensor returns tilt data to correct the shooting angle to obtain accurate vertical and horizontal baselines, and then calculates the actual tilt angle of the tower by combining the tilt angle of the geometric centerline of the tower profile with the triangle method, thus improving the accuracy of the measurement results.

\section{CONCLUSION}

In view of the complicated fault conditions of transmission towers, many interference factors and the difficulty in solving the problem of line selection protection, this paper puts forward the operation status monitoring of transmission towers in power system based on data fusion. Using intelligent monitoring master station, the stability monitoring system of transmission tower is established, which effectively realizes the network monitoring of transmission tower condition. The actual tilt angle of the tower can be accurately obtained by collecting two images of the transmission tower on a specific geometric relationship and calculating the tilt angle of the geometric center line of the tower outline respectively. Transmission towers are finely divided. On the basis of dividing the tower, the bird's nest on the transmission tower can be detected accurately by modeling the color of the bird's nest.

This topic has systematically completed the research on the operation status monitoring of transmission towers in power system based on data fusion, but there are still some shortcomings in this method. To be applied to engineering practice, the following aspects need to be further studied: (1) Further research on the identification method of key components in aerial images of transmission lines, so that these key components can be accurately extracted under different complex backgrounds, and the robustness of the algorithm can be enhanced. (2) Combining theory with practice better, considering the equipment state in different environments and conditions in the algorithm, making the detection results more accurate, and further improving and engineering the method in this paper.

\section{ACKNOWLEDGMENT}

This research work was supported by Chongqing Key Laboratory of Geological Environment Monitoring and Disaster Early-Warning in Three Gorges Reservoir Area, Chongqing Three Gorges University MP2020B0204, the Science \& Technology Research Program of Chongqing Municipal Education Commission.

\section{REFERENCE}

[1] Zhang X, Zhao Y, Zhou L, et al. Transmission Tower Tilt Monitoring System Using Low-Power Wide-Area Network Technology. IEEE Sensors Journal, 2020, PP(99):1-1.

[2] Nan Y, Xie W, Min L, et al. Real-Time Monitoring of Wind-Induced Vibration of High-Voltage Transmission Tower Using an Optical Fiber Sensing System. IEEE Transactions on Instrumentation and Measurement, 2019, PP(99):1-7.

[3] Zhang L, Ruan J, Du Z, et al. Short-Term Failure Warning for Transmission Tower Under Land Subsidence Condition. IEEE Access, 2020, PP(99):1-1.

[4] Gao D W, Wang Q, Zhang F, et al. Application of AI techniques in monitoring and operation of power systems. Frontiers in Energy, 2019, 13(1):71-85.

[5] Huang X, Zhao L, Chen Z, et al. An online monitoring technology of tower foundation deformation of transmission lines. Structural Health Monitoring, 2019, 18(3):949-962.

[6] Ding S, Cheng Y, Zheng H, et al. Uplift Bearing Capacity of Transmission Tower Foundation in Reinforced Aeolian Sand Using Simplified Model Tests. Advances in Civil Engineering, 2021, 2021(58):1-13.

[7] Zhang J, Du X, Xiao W, et al. Condition Monitoring the Health Status of Forced Air Cooling System Using the Natural Frequency of Thermal Network. IEEE Transactions on Power Electronics, 2019, 34(11):10408-10413.

[8] Zhao J, Kamwa I. Guest Editorial: Next Generation of Synchrophasor-based Power System Monitoring, 
Operation and Control. IET Generation Transmission \& Distribution, 2020, 14(19):3943-3944.

[9] Zheng H, Wang Y. Design and Health Evaluation of Monitoring Test Platform for Metro Traction Power Supply System. Advances in Civil Engineering, 2021, 2021(2):1-11.

[10] Zhou C, Liu Y, Wu J, et al. Optimal Sensor Placement and Minimum Number Selection of Sensors for Health Monitoring of Transmission Towers. Shock and Vibration, 2020, 2020(1):1-12.

[11] Han J, Zhang H, Lin D, et al. Evaluation on Safety Performance of Power Transmission Iron Tower Based on Stress Monitoring. E3S Web of Conferences, 2020, 145(4):02084.

[12] Deng F, Zuo P, Wen K, et al. Low Delay Technology Research of Transmission Line Tower Monitoring Network Integrating WSN and RFID. IEEE Access, 2019, PP(99):1-1.

[13] TiAn L, Zhou M, Qiu C, et al. Seismic response control of transmission tower-line system using SMA-based TMD. Structural Engineering \& Mechanics, 2020, 74(1):129-143.

[14] Tian L, Pan H, Ruisheng M A, et al. Seismic failure analysis and safety assessment of an extremely long-span transmission tower-line system. Structural Engineering \& Mechanics, 2019, 71(3):305-315.

[15] Gao Zhengping, Huang Shijun, Huang Fenghua, et al. Safe wind speed for hoisting components and cross-arm of the large span transmission tower. Journal of Nanjing University of Technology, 2019, 041(002):206-211.

[16] Tian L, Liu J, Qiu C, et al. Temperature effect on seismic behavior of transmission tower-line system equipped with SMA-TMD. Smart Structures and Systems, 2019, 24(1):1-14.

[17] Hamzah N H, F Usman. Geospatial analysis of wind velocity to determine wind loading on transmission tower. Wind \& Structures, 2019, 28(6):381-388.

[18] Buryakov G, Sysoeva E. Monitoring of Russian banking system in terms of digital transformation. E3S Web of Conferences, 2021, 273(1):08087.

[19] Dygalo V, Keller A, Evtiukov S. Monitoring of vehicles' active safety systems in operation. Transportation Research Procedia, 2020, 50:113-120.

[20] Zeng T, Yang X, Wan Y, et al. Effectiveness assessment of improvement measures in physical protection system monitoring center. Kerntechnik, 2021, 86(1):33-38.

[21] Li, Peiming, Xiao, et al. Establishment of a Quality Assurance System in the Online Monitoring of Electromagnetic Environment of Alternating Current Power Transmission and Transformation Facilities. Meteorological and Environmental Research, 2019, 10(05):50-54.

[22] Kekalo A, Nemchenko V, Filippov A. Injuriousness of Blumeria graminis and Pyrenophora tritici-repentis in wheat crops and measures of its operation monitoring. E3S Web of Conferences, 2020, 176(4):04008.
Creative Commons Attribution License $\mathbf{4 . 0}$ (Attribution 4.0 International, CC BY 4.0)

This article is published under the terms of the Creative Commons Attribution License 4.0

https://creativecommons.org/licenses/by/4.0/deed.en_US 